\title{
Considerations for PrEP introduction to adolescent girls and young women: Implementation science research in Tanzania
}

Population Council

Follow this and additional works at: https://knowledgecommons.popcouncil.org/departments_sbsr-hiv

Part of the Demography, Population, and Ecology Commons, Family, Life Course, and Society Commons, International Public Health Commons, Medicine and Health Commons, and the Women's Health Commons How does access to this work benefit you? Let us know!

\section{Recommended Citation}

Population Council. 2017. "Considerations for PrEP introduction to adolescent girls and young women: Implementation science research in Tanzania," Activity brief. Washington, DC: Population Council. 


\title{
CONSIDERATIONS FOR PREP INTRODUCTION TO ADOLESCENT GIRLS AND YOUNG WOMEN
}

\author{
IMPLEMENTATION SCIENCE RESEARCH IN TANZANIA
}

Tanzania has made great strides in controlling the HIV epidemic in the last decade, surpassing the national coverage target for voluntary medical male circumcision and reducing new HIV infections among adults by more than 20 percent between 2010 and $2015 .{ }^{1}$ However, the severity of the epidemic varies considerably across sub-populations as well as across and within districts.

Adolescent girls and young women (AGYW) aged 15-24 continue to have high rates of HIV in Tanzania, with HIV prevalence twice that of their male counterparts. ${ }^{2}$ Further, HIV prevalence is 12.6 percent among AGYW who engage in sex work and 11 percent among AGYW who are separated, divorced, or widowed.

AGYW in Tanzania, as in many countries, are particularly vulnerable to HIV due to a host of reasons, including biological (e.g., co-infection with sexually transmitted infections); behavioral (e.g., older sexual partners, inconsistent condom use); societal (e.g., gender inequality); and structur-

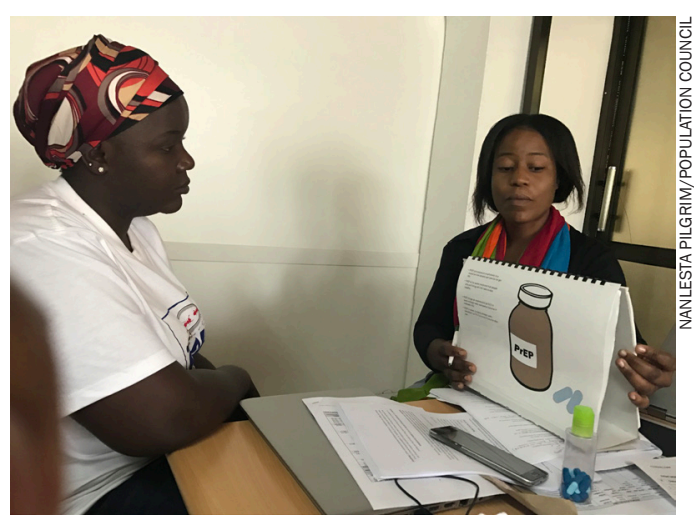

There is a need to carefully examine the individual, interpersonal, structural, and sociocultural factors that may influence PrEP acceptability, uptake, and adherence among AGYW. al (e.g., poverty, lack of access to education).

One option to prevent AGYW from acquiring HIV that is gaining interest and momentum is oral pre-exposure prophylaxis (PrEP). The use of antiretroviral medications by HIV-uninfected persons to prevent getting HIV is a new biomedical intervention that has the potential to substantially reduce HIV acquisition among AGYW within the context of a comprehensive HIV prevention package, if they are able to effectively access and use it.

Many questions remain surrounding the rollout of PrEP for AGYW in Tanzania and other high HIV burden countries. There is need to carefully examine the individual, interpersonal, structural, and sociocultural factors that may influence PrEP acceptability, uptake, and adherence among AGYW. More information is urgently needed to guide policymakers and program managers in determining whether and how to introduce PrEP, how to support informed choice and effective use of PrEP, how to ensure the provision of quality services, and what strategies should be used to address implementation challenges. ${ }^{3}$

In collaboration with the National AIDS Control Programme (NACP) and CSK Research Solutions Ltd, a Tanzania-based research organization, the Population Council is conducting implementation science research in Tanzania to build needed evidence to inform decision-making around PrEP introduction to AGYW, ages 15-24. Learnings from this study and other observational studies and demonstration trials in progress across sub-Saharan Africa, including in Tanzania, will be valuable for informing ongoing programming in Tanzania and the region, as well as HIV prevention efforts for AGYW globally. ${ }^{4}$

Research Partners: CSK Research

Solutions Ltd

Location: Dar es Salaam and Mbeya Districts

Study Duration: 2016-2018

Funder: Bill \& Melinda Gates Foundation

For more information, contact Nanlesta Pilgrim (npilgrim@popcouncil.org), Lou Apicella (lapicella@popcouncil. org), or Catherine Kahabuka (ckahabuka@cskresearch.com). 


\section{POPULATION COUNCIL'S DREAMS IMPLEMENTATION RESEARCH}

This study is part of a portfolio of implementation science studies the Population Council is conducting, with funding from the Bill \& Melinda Gates Foundation, related to the DREAMS (Determined, Resilient, Empowered AIDS-free, Mentored, and Safe) Partnership. DREAMS aims to significantly reduce new HIV infections among AGYW in 10 countries in sub-Saharan Africa, including Tanzania. Our studies are exploring whether the intended beneficiaries-AGYW and their male partners-are being reached and retained in services, how different intervention components are being delivered to each group, and ultimately whether HIV risk among AGYW is being reduced.

\section{OUR RESEARCH}

This study will assess the acceptability of PrEP among AGYW, providers, and key community members; determine the feasibility of and opportunities for providing PrEP to AGYW; and identify key facilitators and barriers to PrEP introduction to AGYW. We will use quantitative and qualitative research methods to generate data on:

- AGYW's knowledge, perceptions, and attitudes toward PrEP; barriers to acceptability, uptake and adherence; and strategies for introducing PrEP. Partnered AGYW, female sex workers, and AGYW engaged in DREAMS programming will be the study sub-groups.

- Male attitudes, perceptions, and concerns about their partner's use of PrEP; and norms and decision-making processes within relationships that might affect AGYW's decision to accept PrEP.

- Parents' and guardians' perceptions of and concerns about PrEP and their attitudes toward providing consent for minor adolescent girls to take PrEP.

- Health care providers' knowledge, perceptions, and preparedness for providing PrEP to AGYW as well as their views of the barriers and facilitators associated with the integration of PrEP into existing services.

- Policy makers' knowledge, perceptions and attitudes toward PrEP for AGYW and key questions and issues that must be addressed to inform decisions regarding introducing PrEP to AGYW.

\section{RESEARCH UTILIZATION}

We will actively engage key stakeholders throughout the study, including gaining their insights on key study questions, interpreting the findings, crafting initial programming recommendations based on findings, and supporting their adoption in Tanzania and beyond. Stakeholders in Tanzania include, among others, the Ministry of Health, Community Development, Gender, Elderly and Children, the National AIDS control Programme; Tanzania Commission for AIDS; regional and district medical officers and AIDS control coordinators; and youth and AIDS service organizations such as SAUTI, TUNAJALI, Tanzania Youth Alliance, and Kihumbe.

\section{WHAT WILL WE LEARN?}

This timely implementation science study will generate important insights from key groups who are likely to play a significant role in PrEP introduction and acceptability among AGYW in both urban and rural areas. Specifically, the study will:

- Identify the factors that are most likely to influence AGYW's decision to take PrEP.

- Identify strategies to generate demand for PrEP among AGYW.

- Examine the degree of influence partners, families, providers, and communities have over PrEP acceptance by AGYW.

- Identify the training and support health providers need to provide PrEP.

- Identify appropriate service delivery settings to offer PrEP.

${ }^{1}$ UNAIDS. 2016. Prevention Gap Report. Geneva: UNAIDS.

${ }^{2}$ National AIDS Control Programme (NACP). 2013. HIV Biological and Behavioral Survey: Tanzania 2013-Female Sex Workers in Seven Regions: Dar es Salaam, Iringa, Mbeya, Mwanza, Tabora, Shinyanga, and Mara (draft). National AIDS Control Programme, Ministry of Health and Social Welfare, and Population Service International (PSI): Dar es Salaam.

${ }^{3}$ Mathur, S, N. Pilgrim, and J. Pulerwitz. 2016. "PrEP introduction for adolescent girls and young women," The Lancet HIV 3: e406-e408.

"PrEP Watch. 2017. “Access basics.” http://www.prepwatch.org/policies-and-programs/access-basics/. Accessed 27 February 2017.
POPULATION COUNCIL

Ideas. Evidence. Impact.
The Population Council confronts critical health and development issues-from stopping the spread of HIV to improving reproductive health and ensuring that young people lead full and productive lives. Through biomedical, social science and public health research in about 50 countries, the Council works with our partners to deliver solutions that lead to more effective policies, programs, and technologies to improve lives worldwide. Established in 1952 and headquartered in New York, the Council is a nongovernmental, nonprofit organization with an international board of trustees. popcouncil.org

Suggested citation: Population Council. 2017. “Considerations for PrEP introduction to adolescent girls and young women: implementation science research in Tanzania," DREAMS Activity Brief. Washington, DC: Population Council. 